\title{
Real-Time Color Image Improvement System for Visual Testing of Nuclear Reactors
}

\author{
Naoki HOSOYA $^{\dagger \text { a) }}$, Nonmember, Atsushi MIYAMOTO ${ }^{\dagger}$, Member $_{\text {, and }}$ Junichiro NAGANUMA ${ }^{\dagger \dagger}$, Nonmember
}

\begin{abstract}
SUMMARY Nuclear power plants require in-vessel inspections for soundness checks and preventive maintenance. One inspection procedure is visual testing (VT), which is based on video images of an underwater camera in a nuclear reactor. However, a lot of noise is superimposed on VT images due to radiation exposure. We propose a technique for improving the quality of those images by image processing that reduces radiation noise and enhances signals. Real-time video processing was achieved by applying the proposed technique with a parallel processing unit. Improving the clarity of VT images will lead to reducing the burden on inspectors. key words: nuclear reactor, visual testing (VT), video image, radiation noise, signal enhancement
\end{abstract}

\section{Introduction}

In nuclear power plants, in-vessel testing is periodically conducted to check for soundness or for preventive maintenance [1]. One inspection procedure is visual testing (VT), which is based on video images taken from an underwater camera [2]. If defect candidates are found in VT, detailed inspections are conducted, such as ultrasonic testing and eddy current testing [3], [4]. Therefore, VT images with high clarity are required for reliable inspection. However, a lot of noise is superimposed on VT images due to radiation exposure. To shield images from radiation, the camera can be covered with a lead or tungsten case. However, since radiation has high transmission capability, radiation noise is difficult to completely remove.

To overcome this, we propose a technique for improving the quality of VT images by image processing that reduces radiation noise and enhances signals. The VT inspector sequentially determines the presence or absence of defects while moving the camera in the vessel. Therefore, realtime processing is needed to practically use the proposed technique. Real-time video image processing is achieved by implementing the proposed technique in a dedicated processor capable of parallel computation. As a result, it is expected to improve the visibility of the VT image and reduce the burden on camera operators and VT inspectors.

The remainder of the paper is organized as follows. Section 2 introduces the problem statement and outlines the

Manuscript received August 25, 2017.

Manuscript revised December 8, 2017.

Manuscript publicized February 16, 2018.

${ }^{\dagger}$ The authors are with Hitachi Ltd., Yokohama-shi, 244-0817 Japan.

${ }^{\dagger}$ The author is with Hitachi-GE Nuclear Energy Ltd., Hitachishi, 317-0073 Japan.

a) E-mail: naoki.hosoya.bj@ hitachi.com

DOI: 10.1587/transinf.2017MVP0007 image improvement system. Section 3 explains details of the VT image improvement algorithm. Section 4 explains the graphics processing unit (GPU) implementation of the proposed algorithm. Experiments on simulated and real images are carried out in Sect. 5. Finally, we conclude the paper in Sect. 6.

\section{Real-Time Color Image Improvement System}

Figure 1 illustrates the proposed VT image improvement system. Inspectors conduct VT on the basis of the color video images of $720 \times 480$ pixels that are taken by an underwater camera. VT images often contain several hindrances, such as radiation noise and low contrast. This motivated us to improve the clarity of VT images by image processing. Our proposed system includes three functions:

- radiation noise reduction,

- signal enhancement, and

- real-time processing (30 frames/s).

The video images that were conventionally displayed on the monitor directly from a charge-coupled device (CCD) camera are imported into the image improvement system and subjected to image improvement processing. By displaying the video image after the image improvement on the moni-

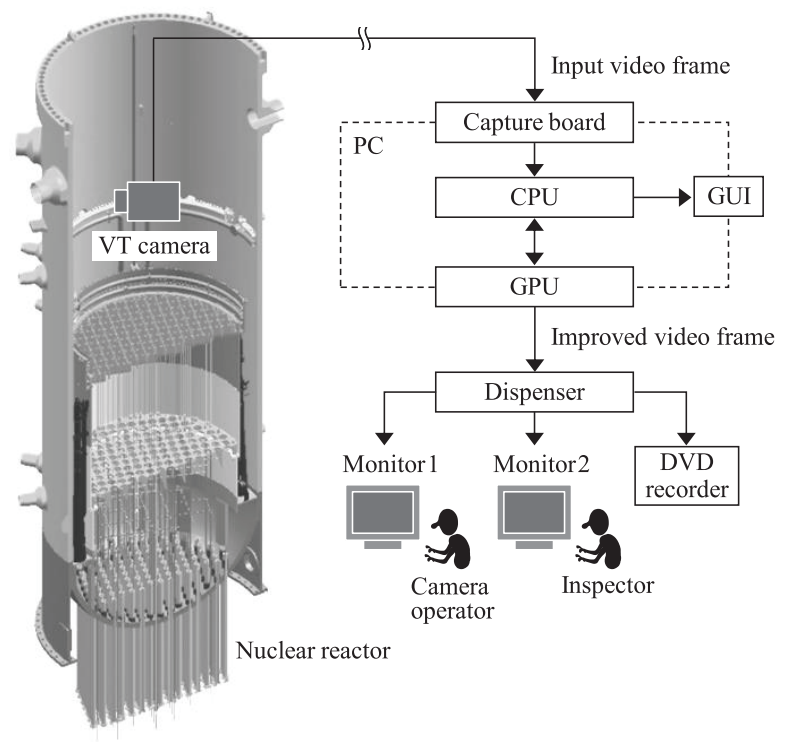

Fig. 1 Video image improvement system. 
tors, the camera operator and the inspector can work effectively on the basis of images with high visibility. In addition, the video image at the time of inspection is recorded on a DVD for history information.

Figure 2 shows the whole processing flow of the image improvement. Since the inner vessel is a radiation field, a lot of radiation noise is superimposed on images. The radiation noise, which is the major cause of visibility deterioration, is reduced by adding time series frames. Other noise such as random noise due to heat is minute compared with radiation noise. In particular, the intensity of radiation noise is large near the reactor core region, and the demand for noise removal is high. In the proposed method, the correspondence between frames is estimated by template matching, and noise is removed by the addition of sequential image frames, also known as a time filter. In order to achieve robust matching between frames that include strong radiation noise, we proposed an automatic removal scheme for unreliable results. The reliability of the matching results is judged from indices concerning the continuity of displacement vector between frames with the peripheral matching results and the uniqueness of the maximum correlation value.

In addition, the reactor's internal structure is complicatedly shaped, and the inspection illumination may not be sufficient in some cases. To improve the inspection accuracy, image contrast emphasis for signal components is conducted. Here, signal components include the defect called a stress corrosion cracking (SCC) and in-vessel structure. With regard to SCC emphasis, only the signal is selectively emphasized using the color information. Generally, there is a risk of emphasizing noise including a lot of high frequency components in signal enhancement. To avoid this problem, a signal component is enhanced after noise has been sufficiently reduced. As it is difficult to completely eliminate noise only by using the aforementioned time filter, a lowpath spatial filter is applied before the signal enhancement. Although the signal component is somewhat blunted by the low pass filter, further image improvement of the input im-

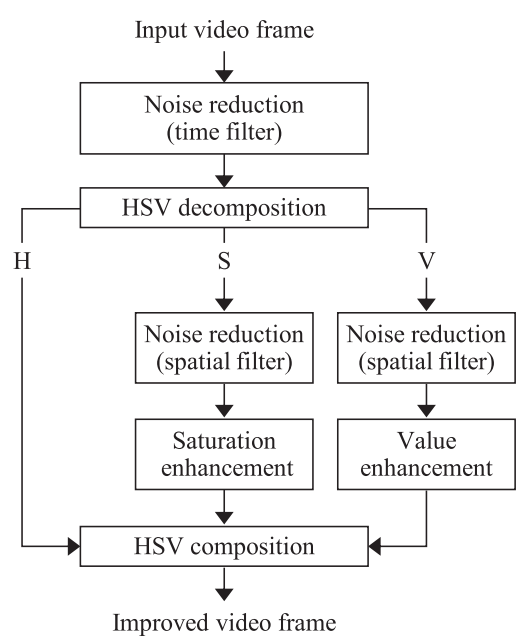

Fig. 2 Overview of processing flow for high image quality. age can be expected by combining the noise reduction with the signal enhancement.

\section{Algorithm}

\subsection{Fast Noise Reduction for Real-Time Processing}

\subsubsection{Time Series Frame Addition}

Noise reduction techniques can be broadly classified into two groups: the spatial filter technique and the time filter technique [5]. Whereas radiation noise components are independent among sequential image frames, signal components have a strong correlation among the frames. We thus decided to use a time filter.

The noise on current frame $f_{n}$ is reduced by addition average among $f_{n}$ and the aligned past frames $\left\{f_{n-k}^{\prime}\right\}$ ( $k=1,2, \cdots, K, K$ : number of past frames). As shown in Fig. 3(b), the current frame $f_{n}$ is divided into multiple rectangular templates $\left\{T_{i}\right\}$ ( $i \in \Omega_{l}, \Omega_{l}=\left\{1,2, \cdots, N_{l}\right\}, N_{l}$ : number of the templates) called "local templates." A displacement vector $\left(\Delta x_{i, k}, \Delta y_{i, k}\right)$ is obtained by matching $f_{n}$ with $f_{n-k}$ for $i$ th template. The aligned past frames are given as $f_{n-k}^{\prime}[x, y]=f_{n-k}\left[x-\Delta x_{i, k}, y-\Delta y_{i, k}\right]$. A noise reduction image $g_{n}$ can be calculated as follows.

$$
\begin{aligned}
g_{n}[x, y]= & M\left(\frac{1}{K} f_{n}[x, y]\right. \\
& \left.+\sum_{k=1}^{K} W_{k}\left(\frac{\operatorname{SAD}_{k}[x, y]}{m[x, y]^{2}}\right) f_{n-k}^{\prime}[x, y]\right),
\end{aligned}
$$

where $M$ is the reciprocal number of the sum of the frame weight, $\operatorname{SAD}_{k}[x, y]$ is the sum of the absolute difference between $f_{n}$ and $f_{n-k}$ in the region near $[x, y], m$ is the standard deviation among the high-pass filtered frames, and $W(a)$ is the monotone decreasing function for a (maximum: $1 / K$, minimum: 0).

The weight $W$ should be set small in the region where the difference in the signal components between frames is large. SAD is changed by not only the difference in but also the quantity of noise. Therefore, $W$ is calculated on the basis of both $\mathrm{SAD}_{k}$ and $m$.

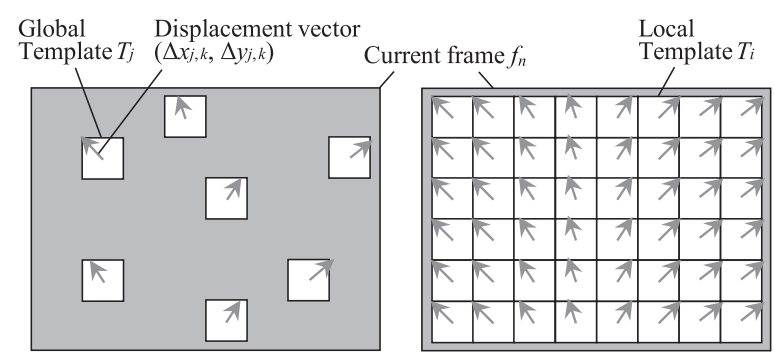

(a) Global matching

(b) Local matching

Fig. 3 Template matching between frames. 


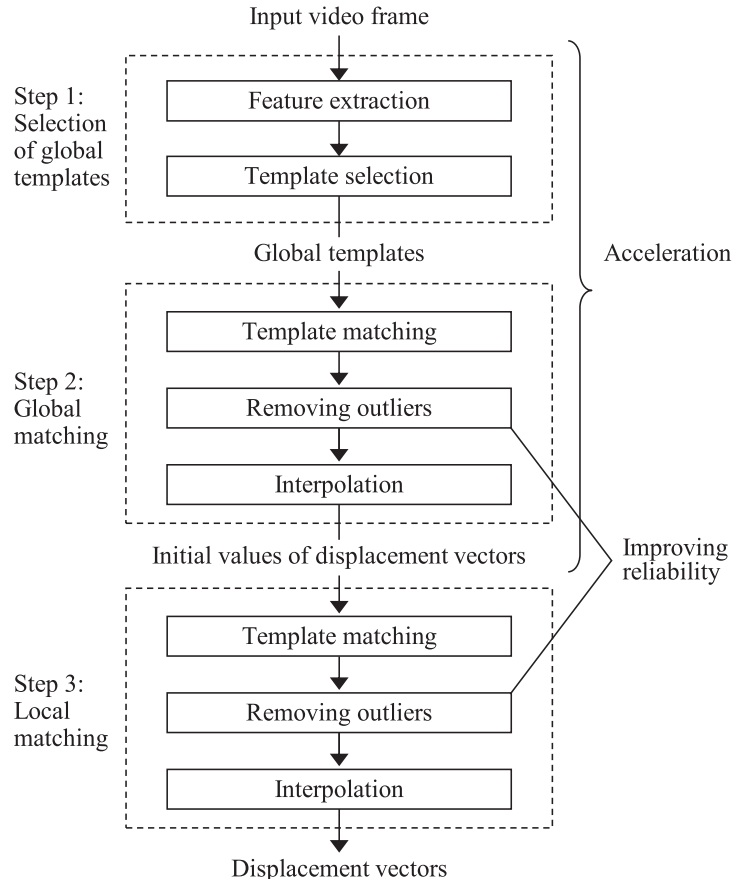

Fig. 4 Fast and reliable template matching.

\subsubsection{Acceleration of Correspondence Search between Frames}

Generally, the computational cost of correlated calculation is high. To speed up the template matching among the frames mentioned in Sect.3.1.1, a multi-stage matching consisting of global and local matching is adopted as shown in Fig. 3 (a) and (b), respectively. The processing flow is explained below with reference to Fig. 4 .

Step 1: Selection of global templates

A few characteristic templates $\left\{T_{i}\right\}\left(i \in \Omega_{g} \subset \Omega_{l}\right)$, called "global templates," are selected from a local template group. The characteristic degrees are evaluated by the amount of edge segments extracted by a Prewitt differential filter [6]:

$$
\begin{aligned}
& \Psi_{x, n}[x, y]=\sum_{i=x+1}^{x+1+\delta} \sum_{j=y-\delta}^{y+\delta} f_{n}[i, j]-\sum_{i=x-1-\delta}^{x-1} \sum_{j=y-\delta}^{y+\delta} f_{n}[i, j], \\
& \Psi_{y, n}[x, y]=\Psi_{x, n}[y, x],
\end{aligned}
$$

where $\delta$ is the parameter for adjusting the size of the edge filter. As this value is set higher, the effect of radiation noise is reduced and more global changes in signal components are evaluated.

The evaluation value $J_{i}$ is calculated for each local template $T_{i}$ on the basis of the edge feature value in Eq. (2) as follows.

$$
\begin{aligned}
J_{i}= & \min \left(\sum_{(x, y) \in \Gamma_{i}} \Psi_{x, n}[x, y], \sum_{(x, y) \in \Gamma_{i}} \Psi_{y, n}[x, y]\right) \\
& +w \sum_{j \in \Omega_{g}} d(i, j)^{2},
\end{aligned}
$$

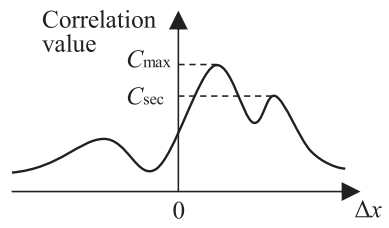

(a) Global matching

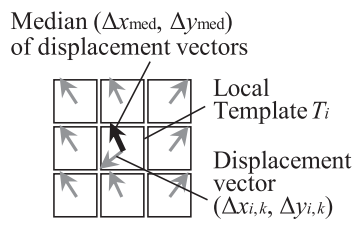

(b) Local matching
Fig. 5 Excluding matching results with low reliability.

where $\Gamma_{i}$ is the coordinate set of the pixels contained in the template $T_{i}, d(i, j)$ is the Euclidean distance between the $i$ and $j$ th local templates, and $w$ is the weight.

The $N_{g}$ templates are extracted as the global templates in descending order of $J_{i}$. After registering the serial number of the extracted template in $\Omega_{g}, J_{i}$ of the remaining templates are updated. The next global template is extracted from the remaining templates sequentially. Equation (3) evaluates two kinds of criteria weighted by $w$. The first term evaluates the edge amount, which is a clue of the matching. A rich edge in the global template is thought to lead to a reliable matching between time series frames. In order to estimate the two-dimensional displacement vector $\left(\Delta x_{i, k}, \Delta y_{i, k}\right)$, the template needs to include enough edge segments in both the $x$ - and $y$-directions. Therefore, the minimum value of the edge amount in the $x$ - and $y$-directions is evaluated. The second term evaluates the distance between global templates. The distribution of displacement vectors between frames can be estimated more accurately by arranging global templates all around within the image.

Step 2: Global matching

As illustrated in Fig. 3 (a), the current frame $f_{n}$ is matched to each past frame $f_{n-k}$ at the positions of each global template $T_{j}$, and the displacement vector $\left(\Delta x_{j, k}, \Delta y_{j, k}\right)$ is estimated. The matching position is searched for within the range of \pm 32 pixels around each template. Template matching may sometimes fail due to image noise in a strong radiation field. To improve the reliability, a robust algorithm is included for removing outlying matching results. In global matching, unreliable vectors satisfying the following condition are removed as shown in Fig. 5 (a).

$$
\frac{C_{\max }-C_{\mathrm{sec}}}{C_{\max }}<p_{1},
$$

where $C_{\max }$ is the maximum correlation value in the search range, and $C_{\mathrm{sec}}$ is the second one, which satisfies the convex condition. That is, $C_{\mathrm{sec}}$ is higher than the correlation values at its neighboring pixels. The variable $p_{1}$ is a parameter.

If the difference between $C_{\max }$ and $C_{\mathrm{sec}}$ is small, the reliability of matching is considered to be low. The dense displacement vectors can be estimated by linear interpolating of $\left(\Delta x_{j, k}, \Delta y_{j, k}\right)$ excluding outliers that do not satisfy the condition of Eq. (4).

Step 3: Local matching

As illustrated in Fig. 3 (b), the current frame $f_{n}$ is matched to each past frame $f_{n-k}$ at the positions of each local template $T_{i}$. The displacement vectors of the local templates can 
be calculated quickly by using the hill-climbing method [7] with adequate initial values, which are estimated in step 2. Similar to global matching, local matching may sometimes fail due to image noise. As illustrated in Fig. 5 (b), outlying vectors are removed and interpolated in accordance with the following equation.

$$
\begin{aligned}
(\Delta x, \Delta y) & =\left\{\begin{array}{ll}
(\Delta x, \Delta y) & \text { if } D<p_{2} \\
\left(\Delta x_{\mathrm{med}}, \Delta y_{\mathrm{med}}\right) & \text { otherwise }
\end{array},\right. \\
D & =\sqrt{\left(\Delta x-\Delta x_{\mathrm{med}}\right)^{2}+\left(\Delta y-\Delta y_{\mathrm{med}}\right)^{2}},
\end{aligned}
$$

where $\left(\Delta x_{\text {med }}, \Delta y_{\text {med }}\right)$ is the median of the displacement vector in the eight-connected neighboring templates, and $p_{2}$ is a parameter.

Unlike global matching, local matching is conducted densely. Assuming the continuity of the displacement vector, judgment and correction of the outlier are performed on the basis of the difference in the tendency with the displacement vector in the neighborhood.

With steps 1-3, the displacement vectors can be estimated with high speed and high reliability. The aligned past frames $\left\{f_{n-k}^{\prime}\right\}$ are given on the basis of the displacement vector.

\subsection{Signal Enhancement Using HSV Information}

Signal components include an SCC, and the in-vessel structure is enhanced by contrast transformation as shown Fig. 2. The SCC is one of the most representative defects to be inspected and is often observed as a slightly vivid crack because of corrosion. Preventive maintenance can be carried out by detecting defects in the early stages of occurrence.

After noise reduction, we convert a red, green, and blue (RGB) color image $g_{n}=(r, g, b)$ into an hue, saturation, and value (HSV) color image $(h, s, v)$ [8]. Since the saturation in the $\mathrm{SCC}$ region is assumed to be high, the high-frequency component of $\mathrm{s}$ is strongly emphasized. Similarly, since the value in the region of the vessel structure is assumed to be high, the high-frequency component of $v$ is emphasized. However, since radiation noise has high intensity, noise is difficult to completely eliminate only by the noise reduction of the time filter technique described in Sect.3.1. To avoid amplifying residual noise on $g_{n}$, a low-path filter is applied to $g_{n}$ before the saturation and signal enhancement. Although the signal component is somewhat blunted by the low pass filter, the input image can be expected to be further improved by combining with the signal enhancement. The output image $g_{\text {sig,n }}$ is

$$
\begin{aligned}
g_{\text {sig }, \mathrm{n}}= & \operatorname{RGB}(h, E(s), E(v)), \\
E(a)=a- & (L(a)-B(L(a))) \\
& +G(L(a)-B(L(a))) .
\end{aligned}
$$

Here, $\mathrm{RGB}()$ is a conversion function from HSV to RGB components. The functions $L(), B(), G()$ are the low-pass filter, bilateral filter [9], and gamma correction.
The basic idea of the formulation in (6) can be explained as follows. The term $L(a)-B(L(a))$ in Eq. (6b) is the high frequency component of the residual noise reduction image. For example, in the signal enhancement processing using a linear shift invariant filter, an unnatural pattern called ringing occurs near the image edge when processing is strongly applied [10]. On the other hand, a bilateral filter is a high performance edge preserving low pass filter with few such side effects. The high frequency component is extracted by the difference in the image before and after bilateral filtering and is selectively emphasized by function $G()$ of gamma correction.

\section{GPU Implementation}

We implemented the proposed algorithm into a parallel processor, that is, a GPU, and improved the real-time display of video images. Table 1 shows the device configuration of the real-time video image improvement system. A relatively compact PC case $(\mathrm{H} 212 \times \mathrm{W} 270 \times \mathrm{D} 393 \mathrm{~mm})$ is used so that it can be easily carried into the inspection space beside the reactor.

The GPU (GeForce GTX 480) has 15 streaming multiprocessors (SMs) consisting of 32 streaming processors (SPs) and a 48k byte shared memory capable of high-speed access in each SM. The computation is divided and implemented in the GPU so that as many operations as possible could be executed in parallel at the same time. As an example of the implementation, the parallelization of global matching explained is illustrated in Fig. 6. There are seven global templates. The $4 \times 7$ thread blocks, each of which includes $64 \times 16$ threads, are allocated to SMs and calculated in parallel. Real-time processing is achieved in conjunction with the acceleration algorithm in Sect.3.1.2 and GPU implementation. Numerical results are shown in Sect. 5.

Table 1 Device configuration.

\begin{tabular}{ll}
\hline Item & Model, Manufacturer \\
\hline PC & Type SLAP, JSC \\
Capture board & Intensity Pro, Blackmagic-Design \\
GPU & GeForce GTX 480, Nvidia \\
\hline
\end{tabular}

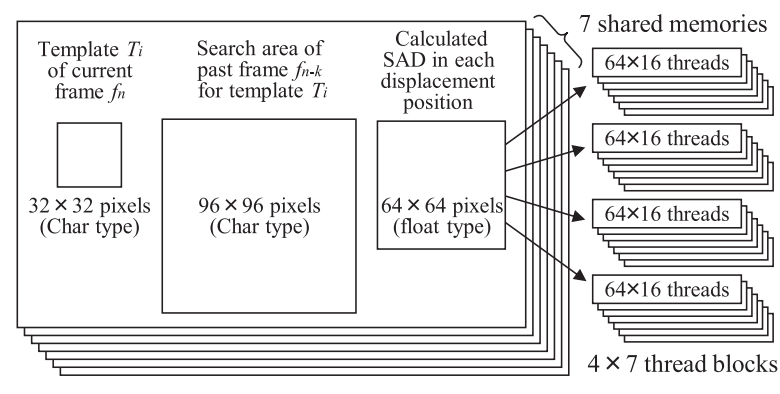

Fig. 6 GPU implementation. 


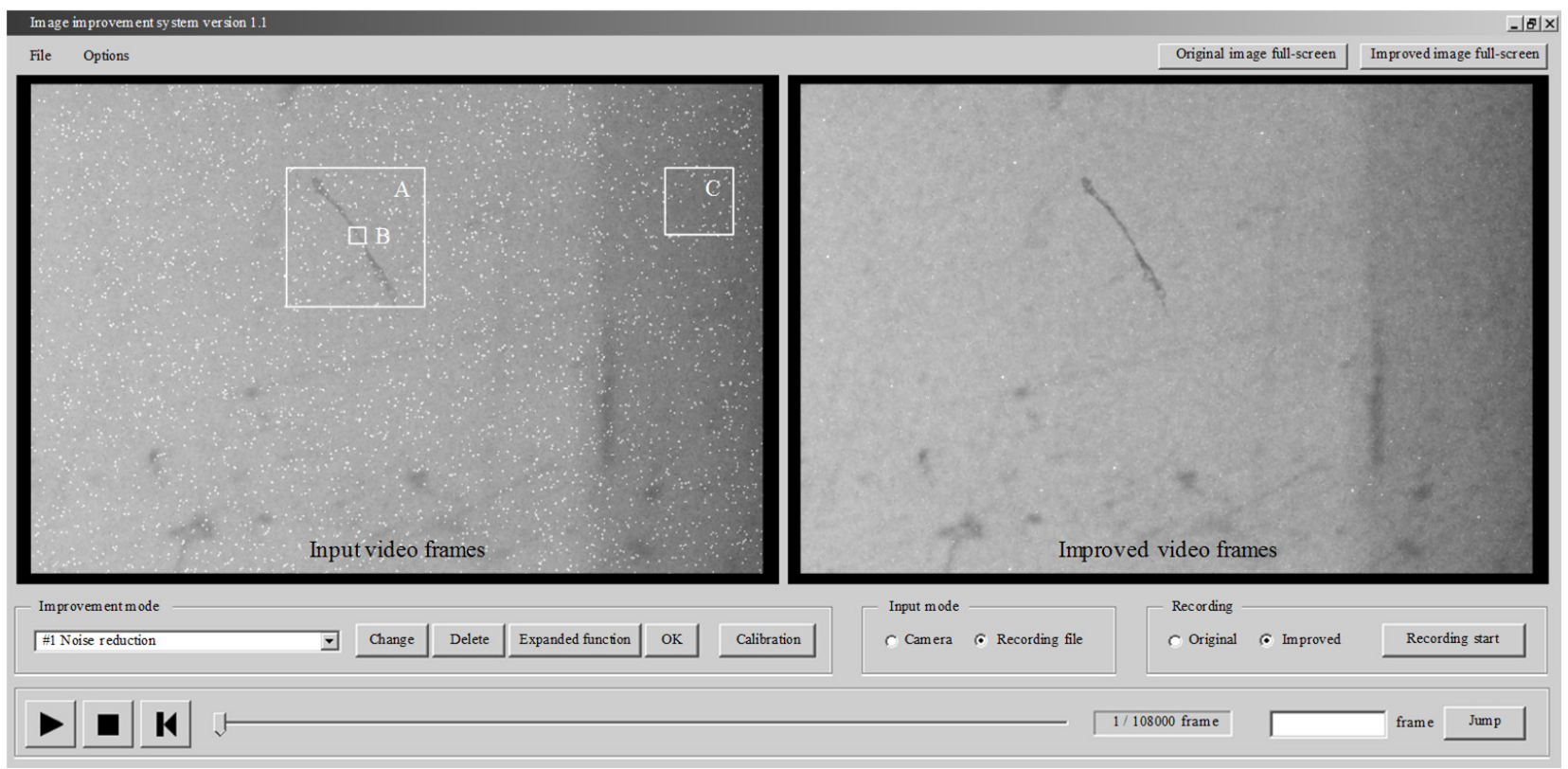

Fig. 7 Screen shot showing result.

Table 2 Experimental conditions.

\begin{tabular}{ll}
\hline Item & Value \\
\hline Image size (pixel) & $720 \times 480$ \\
$K$ : Number of past frames & 6 \\
$N_{g}:$ Number of global templates & 7 \\
$N_{l}:$ Number of local templates & 345 \\
$\delta:$ Y size of edge filter in Eq. (2a) & 4 \\
$p_{1}:$ Parameter of Eq. (4) & 0.1 \\
$p_{2}:$ Parameter of Eq. (5) & 3 \\
\hline
\end{tabular}

\section{Experimental Results}

In this section, we present several experiments on simulated and real video image data. The experimental conditions common to all experiments are listed in Table 2. Here, a supplementary explanation will be given on the processing parameters $\delta, P_{1}, P_{2}$. The parameter $\delta$ is the size of the edge filter, and it is necessary to detect only the signal components such as the edges of the reactor's internal structure. If $\delta$ is too large, a fine signal component can not be detected, and conversely if $\delta$ is too small, noise will be detected. The parameter $P_{1}$ and $P_{2}$ are thresholds for rejecting the failed matching results due to radiation noise. The values were experimentally set, but since the setting values have some flexibility, common values can be given to all experiments.

\subsection{Image Quality Evaluation Using Simulated Images}

We simulated the VT images to evaluate the effectiveness of the proposed technique. It is difficult to theoretically explain the distribution tendency of the CNR improvement ratio in changing the quantity of noise. Therefore, the experiment was conducted by using pseudo VT image for improvement validation. The color filters in the CCD camera are arranged in a Bayer pattern, and the RGB values are provided by adaptive color plane interpolation (ACPI) [11]. From the CCD pixel value $e_{0}$ without radiation noise, the value $e$ with noise is calculated by the following equation.

$$
\begin{gathered}
e[i, j]=\min \left(e_{0}[i, j]+\sum_{\delta i, \delta j} n_{r}[i+\delta i, j+\delta j]\right. \\
\left.\times G\left(\sigma_{n}\right)[\delta i, \delta j], 255\right), \\
n_{r}[i, j]=\left\{\begin{array}{ll}
255 & \text { if }|r|>R \\
0 & \text { otherwise }
\end{array},\right. \\
G\left(\sigma_{n}\right)[i, j]=\exp \left(-\frac{i^{2}+j^{2}}{2 \sigma_{n}^{2}}\right),
\end{gathered}
$$

where $r$ is normally distributed random numbers with a mean of 0.0 and variance of 1.0 , and $R$ is a parameter.

In Eq. (7), it is assumed that one radiation pulse collides with one CCD element. In many cases, the voltage of the CCD element with radiation collision is saturated, and the brightness value increases in the CCD elements around the saturated elements. The arrangement of RGB pixels is given by the Bayer pattern, and the noise distribution given by Eq. (7c). The RGB values are calculated from $e$ by ACPI. As parameter $R$ is set small, a lot of noise is imposed on the simulated image. The minimum value of $R$ is set to a value at which the standard deviation of noise is about 40 pixels. This amount of noise corresponds to the maximum radiation amount assumed in VT (about $1000 \mathrm{~Gy} / \mathrm{h}$ ).

Figure 7 shows screen shots of the result. Input and output images are displayed on the left and right. Figure 8 shows expanded images of region A in Fig. 7, and a crack can be observed as a black line. Figure 8 (a), (b), and (c) are 


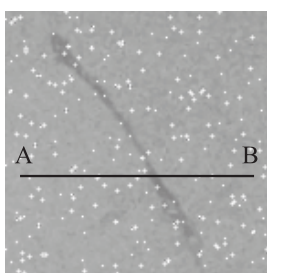

Radiation noise

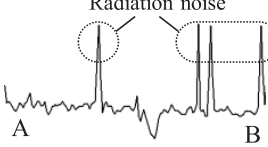

(a) Input image
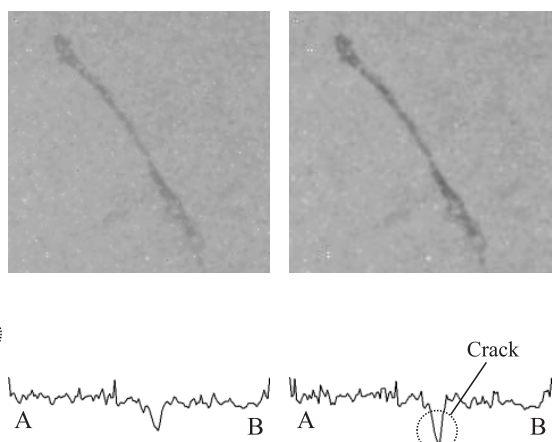

(b) Noise reduction

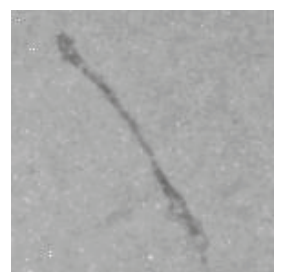

Fig. 8 Enlarged images of region A in Fig. 7.

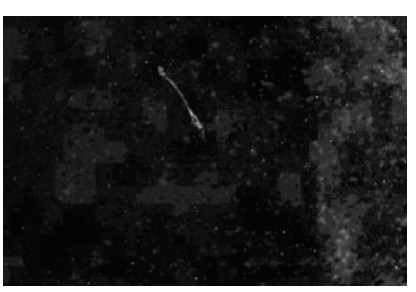

(a) Saturation

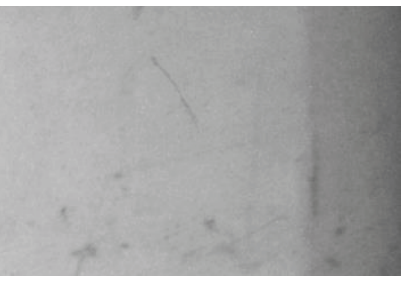

(b) Value
Fig. 9 HSV component images.

the input image, the image with noise reduction proposed in Sect. 3.1, and the image with the signal enhancement proposed in Sect. 3.2. Image profiles along the paths indicated by the line A-B are shown below each image. The proposed method could effectively enhance the crack without noise amplification, improving its visibility.

Figures 9 (a) and (b) are saturation and value components of an HSV image after noise reduction. Since the saturation of the crack seems to be high, the crack can be selectively enhanced in the improved image.

The quality of the improved image is quantitatively evaluated by using the contrast-to-noise ratio (CNR) [12], [13]. Regions B and C in Fig. 7 are used for contrast and noise calculation, respectively. Region $\mathrm{B}$ contains a signal pattern with a large brightness change, and the difference between the 90 th and the 10 th percentile of the brightness value in Region B is defined as the contrast. Region C contains almost no signal pattern, and the noise component is dominant. The standard deviation of the brightness value in Region C is defined as noise. Figure 10 (a) shows the CNR improvement ratio between the input image and improved image for a variation of noise amounts (horizontal axis), which are adjusted by changing $R$ in Eq. (7). Although the effects differ in accordance with the noise amount, the noise reduction and signal enhancement improved CNR up to 1.2-3.4 times and 1.6-4.2 times, respectively. The CNR improvement ratio in Fig. 10 (a) is separated into the contrast improvement ratio and noise reduction ratio in Fig. 10 (b) and (c). As we can see from the change in the noise reduction ratio before and after signal enhancement, noise is properly removed without amplification. Therefore, the ef-

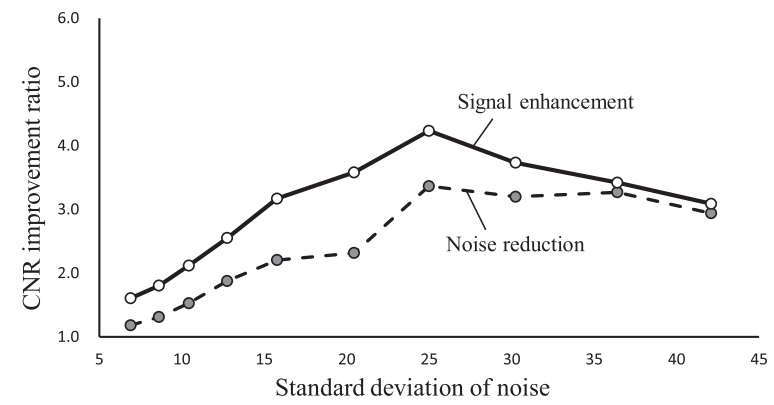

(a) CNR improvement ratio

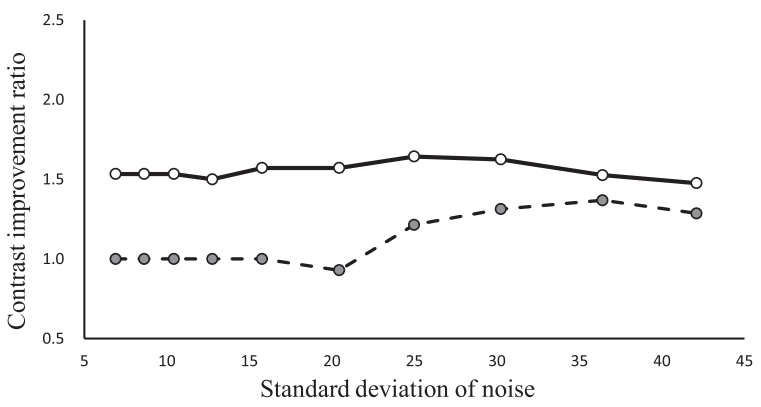

(b) Contrast improvement ratio

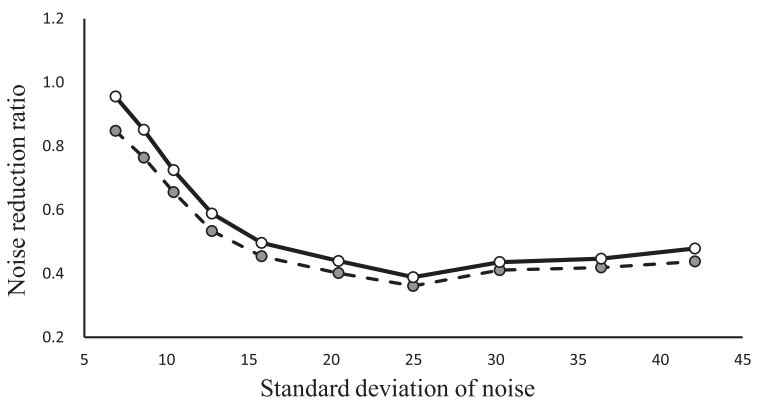

(c) Noise reduction ratio

Fig. 10 CNR improvement ratio between input image and improved image (simulated images).

fect of contrast improvement improves the CNR directly.

\subsection{Image Quality Evaluation Using Real VT Images}

To obtain a clear view of how the proposed method practically improves the VT images, we carried out several experiments using 10 real images taken of each part of the nuclear reactor. Figure 11 shows the image improvement ratio between the input image and improved image similarly to Fig. 10. To compare the simulation and real results, the graphs in Fig. 10 are superimposed on Fig. 11. Since the actual VT images contain variations of the structure and noise shape, the real and simulation results are not completely the same. However, the distribution tendency of the CNR improvement ratio in changing the quantity of noise is roughly similar to that in the simulation in Fig. 11 (a). That is, when the standard deviation of the noise on the horizontal axis is between 5 and 25, as the noise increases, more noise can be removed and the CNR improvement ratio also increases. However, when the standard deviation of the noise exceeds 


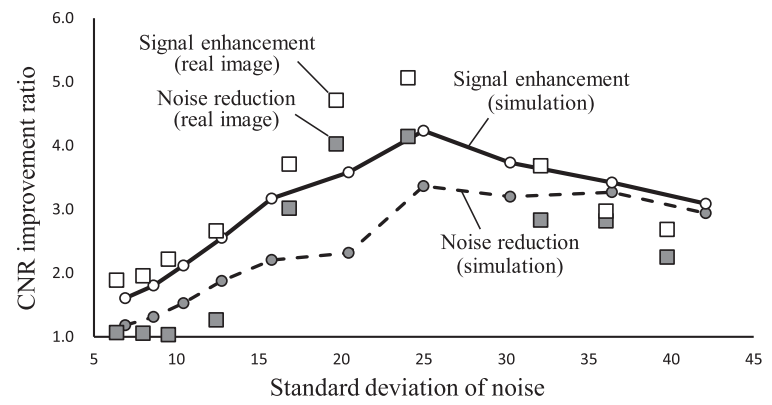

(a) CNR improvement ratio

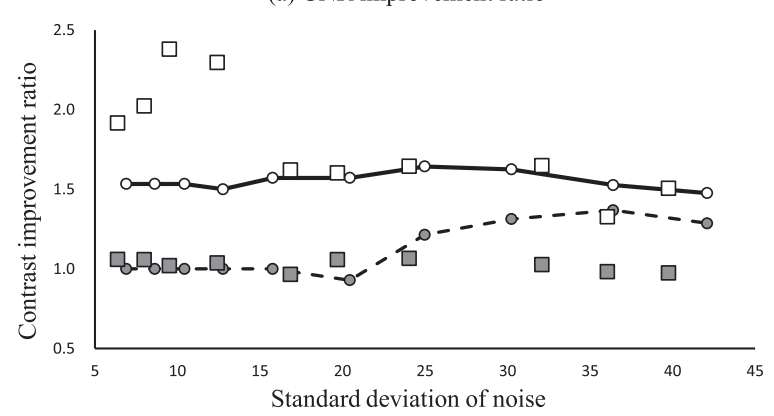

(b) Contrast improvement ratio

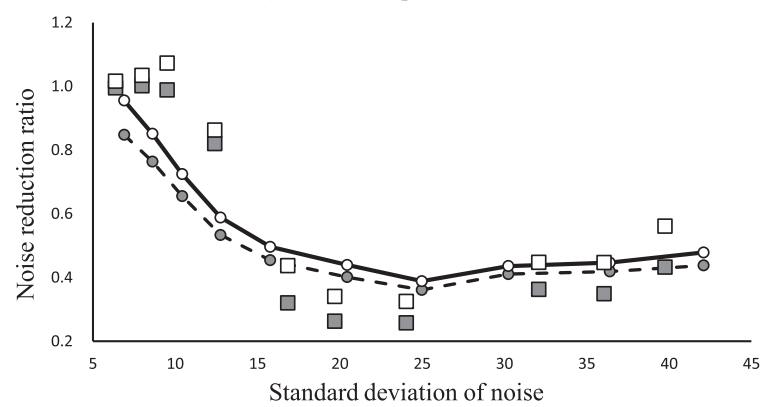

(c) Noise reduction ratio

Fig. 11 CNR improvement ratio between input image and improved image (real images).

about 25 , the limit of reducing noise by adding frames gradually appears, and the CNR improvement ratio becomes low. As we can see from Fig. 11 (c), the noise is not amplified before or after signal enhancement, similar to the simulation. As a result, the noise reduction and signal enhancement improved CNR up to 1.0-4.1 and 1.9-5.1 times in Fig. 11 (a), respectively.

\subsection{Processing Time}

Figure 12 shows the processing time of image improvement for one frame. To display 30 frames per second in real time, the processing time per frame needs to be less than 0.033 seconds. The acceleration algorithm proposed in Sect. 3.1.2 considerably reduced the processing cost of correspondence search, which accounts for most of the processing time. The total processing time in the CPU is reduced from 31 to 4 seconds (left and middle bars in Fig. 12). Furthermore, the parallelization done by using a GPU shortened the processing time to 0.023 seconds (right bar in Fig. 12), resulting in real-time display.

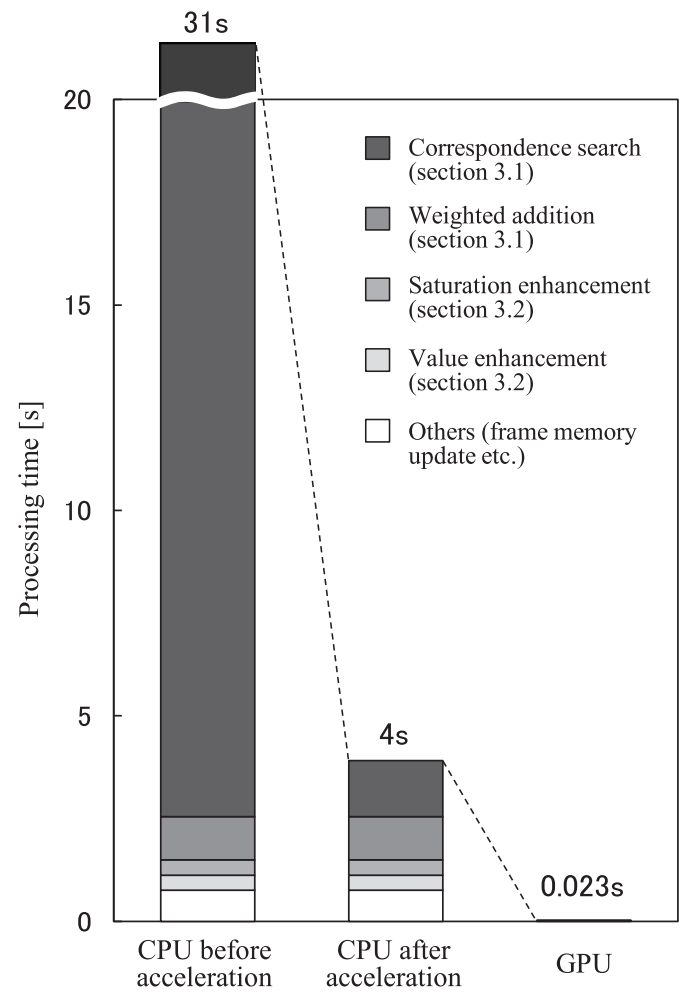

Fig. 12 Processing time.

\section{Conclusion and Future Work}

Nuclear power plants require highly reliable visual testing (VT). In this study, we developed a real-time color image improvement system for VT in nuclear reactors. We proposed robust and fast image processing to improve the quality of low signal-to-noise VT images, which can suffer from strong radiation effects. Experimental results demonstrated the significance of the proposed method. The contrast-tonoise ratio (CNR) is improved 1.9-5.1 times in real images in real time: processing time per frame is 0.023 seconds. The improving visibility of VT images is expected to lead to reducing the burden on inspectors.

For future work, the effectiveness of our system will need to be continuously verified in real reactors. The practicality of using the technique to check soundness or for preventive maintenance may be promoted.

\section{References}

[1] T. Ikegami, T. Shimura, and M. Koike, "Plant life management and maintenance technologies for nuclear power plants," Hitachi Review, vol.50, no.3, pp.84-88, 2001.

[2] M. Odakura, M. Kometani, M. Koike, M. Tooma, and Y. Nagashima, "Advanced inspection technologies for nuclear power plants," Hitachi Review, vol.58, no.2, pp.82-87, 2009.

[3] I. Komura, T. Hirasawa, S. Nagai, J. Takabayashi, and K. Naruse, "Crack detection and sizing technique by ultrasonic and electromagnetic methods," Nuclear Engineering and Design, vol.206, no.2-3, pp.351-362, 2001.

[4] S. Kitazawa, M. Odakura, K. Otani, and Y. Adachi, "Advanced in- 
spection technologies for energy infrastructure," Hitachi Review, vol.59, no.3, pp.111-115, 2010.

[5] A. Rosenfeld and A.C. Kak, Digital Picture Processing, Academic Press, 1976.

[6] B.S. Lipkin and A. Rosenfeld, Picture Processing and Psychopictorics, Academic Press, 1970.

[7] B.D. Lucas and T. Kanade, "An iterative image registration technique with an application to stereo vision," Proc. Imaging Understanding Workshop, pp.121-130, 1981.

[8] A.R. Smith, "Color Gamut Transform Pairs," Proc. Conf. on ACMSIGGRAPH, vol.78, pp.12-18, 1978 .

[9] C. Tomasi and R. Manduchi, "Bilateral filtering for gray and color images," Proc. IEEE Int. Conf. on Computer Vision, pp.836-846, 1998.

[10] J.G.M. Schavemaker, M.J.T. Reinders, J.J. Gerbrands, and E. Backer, "Image sharpening by morphological filtering," Pattern Recognition, vol.33, no.6, pp.997-1012, 2000.

[11] R. Ramanath, W.E. Snyder, G.L. Bilbro, and W.A. Sander, "Demosaicking methods for bayer color arrays," J. Electronic Imaging, vol.11, no.3, pp.306-315, 2002.

[12] D. Shao and D.C. Liu, "Local histogram matching based bilateral filter to ultrasound speckle reduction," Proc. 5th IEEE Int. Conf. Bioinformatics and Biomedical Engineering, pp.1-4, 2011.

[13] A.E. Petropoulos, G.F. Vlachopoulos, S.G. Skiadopoulos, A.N. Karahaliou, and L.I. Costaridou, "Improving image quality in dual energy CT by edge-enhancing diffusion denoising," Proc. 13th IEEE Int. Conf. Bioinformatics and Bioengineering, pp.1-4, 2013.

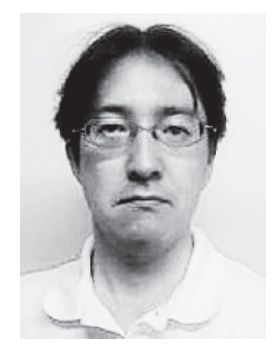

Naoki Hosoya received a B.S. degree in control engineering from Tokyo Institute of Technology in 1989. He is currently a senior researcher at R\&D Group, Hitachi, Ltd. His research interests include image processing, pattern recognition, and inspection system.

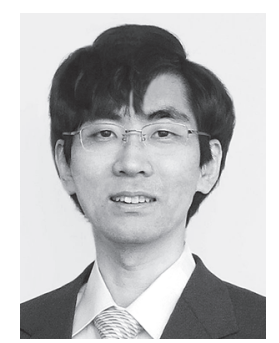

Atsushi Miyamoto received a B.S. degree in precision engineering and M.S. and Ph.D. degrees in system information engineering from Hokkaido University in 1996, 1998, and 2001. $\mathrm{He}$ is currently a senior researcher at R\&D Group, Hitachi, Ltd. His research interests include robust image processing and statistical pattern recognition. He is a member of IEICE, JSPE, IEEJ, IIEEJ, JAMIT and IEEE.

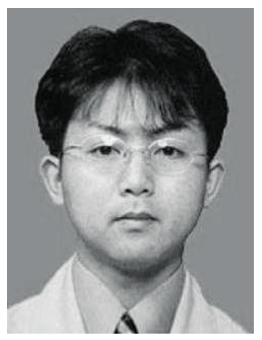

Junichiro Naganuma received a B.S. degree in mechanical engineering from National Institute of Technology, Ibaraki College in 1994. $\mathrm{He}$ is currently an engineer at Hitachi-GE Nuclear Energy, Ltd. He works on the development of non-destructive inspection technology. 